\title{
Novel Three Order Methods for Solving a System of Nonlinear Equations
}

\author{
M.Q. Khirallah and M.A. Hafiz* \\ Department of mathematics, Faculty of Science and Arts, Najran University, Najran 1988, \\ Saudi Arabia
}

Keywords: System of Non-Linear Equations (SNLE); Predictor-Corrector methods; quadrature formulas.

Abstract: In this paper, we suggest and study Simpson's formula, and Newton's two, three and four Cosed formulas iterative methods for solving the system of nonlinear equations by using PredictorCorrector of Newton method. We present four new algorithms for solving the system of nonlinear equations (SNLE). We prove that these new algorithms have convergence. Several numerical examples are given to illustrate the efficiency and performance of the new iterative methods. These new algorithms may be viewed as an extensions and generalizations of the existing methods for solving the system of nonlinear equations.

\section{Introduction}

Recently, several iterative methods have been used to solve nonlinear equations and the system of nonlinear equations (SNLE) [1-6]. Ali et. al. [7] have developed a new predictorcorrector method for solving nonlinear equation by using the weight combination of the midpoint, and Trapezoidal quadrature formulas. Wang [8], using a third order family of Newton-Like iteration method for solving nonlinear equations; Noor et.al. [9] have considered an alternative decomposition technique which does not involve the derivative of the domain polynomial. Chun [10] has presented a new iterative method to solving nonlinear equations by using $\mathrm{A}$ domain decomposition. Yong et.al. [11] developed a new scheme for the construction of iterative methods for the solution of nonlinear equations and giving a new class of methods from any iterative method. Furthermore, several iterative methods have been developed for solving the system of nonlinear equations (SNLE) by using various techniques such as Newton's method, Revised Adomian decomposition method, homotopy perturbation method, Householder iterative method [12-18]. Darvishi et.al. [19-20] have constructed two new methods. The two methods are third order Newton type method and super cubic iterative method for solving (SNLE), H, He [21] and Golbabai et. al. [22] have applied the homotopy perturbation method to build a new family of Newton-like iterative methods for solving (SNLE). Mustafa [23] has considered a new decomposition method for solving (SNLE). Jank et.al. [24] developed two families of third-order methods for solving (SNLE). Finally, Hueso [25] presented a family of predictor-corrector methods free from second derivative for solving (SNLE).

In this paper, we aim to generalize and apply Simpson's formula, and Newton's two, three and four Cosed formulas to give four new algorithms for solving (SNLE). Finally, some numerical examples have been fulfilled with Maple software to demonstrate our method and comparison of our results with those derived from previous methods. All test problems reveal accuracy and fast convergence of our method.

\section{System of Nonlinear Equations(SNLE)}

The general form of a system of non-linear equations is

$$
\begin{gathered}
f_{1}\left(x_{1}, x_{2}, \cdots, x_{n}\right)=0, \\
f_{2}\left(x_{1}, x_{2}, \cdots, x_{n}\right)=0, \\
\vdots \\
f_{n}\left(x_{1}, x_{2}, \cdots, x_{n}\right)=0,
\end{gathered}
$$


where each function i $\mathrm{f}$ can be thought of as mapping a vector $\mathrm{x}=\left(x_{1}, x_{2}, \ldots, x_{\mathrm{n}}\right)$ of the ndimensional space $\mathrm{R}^{\mathrm{n}}$, into the real line $\mathrm{R}$. The system can alternatively be represented by defining a functional $F$, mapping $R^{n}$ into $R^{n}$ by :

$$
F\left(x_{1}, x_{2}, \ldots, x_{\mathrm{n}}\right)=f_{l}\left(x_{1}, x_{2}, \ldots, x_{\mathrm{n}}\right), \ldots, f_{n}\left(x_{1}, x_{2}, \ldots, x_{\mathrm{n}}\right)^{T}
$$

Using vector notation to represent the variables $x_{1}, x_{2}, \ldots, x_{\mathrm{n}}$ a system (1) can be written as the form:

$$
F(\mathrm{x})=0
$$

The functions $f_{1}, f_{2}, \ldots, f_{n}$ are called the coordinate functions of $\mathrm{F}$ [26].

\section{Iterative methods}

Suppose that $X$ be the simple zero of sufficiently differentiable functions and consider the numerical solution of the system of equations $F(\mathrm{x})=0$, where $F: D \subseteq R^{n} \rightarrow^{n}$ is a smooth mapping that has continuous second order partial derivatives on a convex open set $\mathrm{D}$, and that has a locally unique root $\mathrm{x}$ in $\mathrm{D}, F(\mathrm{x})=\left(f_{1}(\mathrm{x}), f_{2}(\mathrm{x}), \ldots, f_{n}(\mathrm{x})\right)^{T}, \mathrm{x}=\left(x_{1}, x_{2}, \ldots, x_{\mathrm{n}}\right)^{T}$ and $f_{i}: R^{n}$ is a nonlinear function, then,

$$
F(\mathrm{x})=F\left(\mathrm{x}_{i}\right)+\int_{x_{i}}^{x} F^{\prime}(t) d t
$$

If we approximate the integral in equation (3) by using Simpson's methods formula for solving a definite integral $\int_{x_{i}}^{x} F^{\prime}(t) d t$, are as follows [2], then

$$
\int_{\mathrm{x}_{i}}^{\mathrm{x}} F^{\prime}(t) d t=\left[F^{\prime}\left(\mathrm{x}_{i}\right)+4 F^{\prime}\left(\frac{\mathrm{x}_{i}+\mathrm{x}}{2}\right)+F^{\prime}(\mathrm{x})\right] \frac{\left(\mathrm{x}-\mathrm{x}_{i}\right)}{3}
$$

From (3) and (4), we have,

$$
F(\mathrm{x})=F\left(\mathrm{x}_{i}\right)+\left[F^{\prime}\left(\mathrm{x}_{i}\right)+4 F^{\prime}\left(\frac{\mathrm{x}_{i}+\mathrm{x}}{2}\right)+F^{\prime}(\mathrm{x})\right] \frac{\left(\mathrm{x}-\mathrm{x}_{i}\right)}{3}
$$

Since, $F(\mathrm{x})=0$ then

$$
\mathrm{x}=\mathrm{x}_{i}-6\left[F^{\prime}\left(\mathrm{x}_{i}\right)+4 F^{\prime}\left(\frac{\mathrm{x}_{i}+\mathrm{x}}{2}\right)+F^{\prime}(\mathrm{x})\right]^{-1} F\left(\mathrm{x}_{i}\right)
$$

which is an implicit method. To overcome this drawback, one usually uses the prediction and correction technique. With the formulation (6) and with selecting Predictor-Corrector of Newton method we will have followed a two-step iterative method for solving the system of nonlinear equation (2) as follows

Algorithm 1: For a given $\mathrm{X}_{0}$, compute the approximate solution $\mathrm{X}_{i+1}$ by iterative scheme.

$$
\mathrm{y}_{i}=\mathrm{x}_{i}-J^{-1}\left(\mathrm{x}_{i}\right) F\left(\mathrm{x}_{i}\right) \text {, }
$$

$$
\mathrm{x}_{\mathrm{i}+1}=\mathrm{x}_{i}-6\left[F^{\prime}\left(\mathrm{x}_{\mathrm{i}}\right)+4 F^{\prime}\left(\frac{\mathrm{x}_{i}+\mathrm{y}_{\mathrm{i}}}{2}\right)+F^{\prime}\left(\mathrm{y}_{\mathrm{i}}\right)\right]^{-1} F\left(\mathrm{x}_{i}\right)
$$

We can also approximate the integral in equation(3) by using another three point Newton-Cosed, formula [27], then

$$
\int_{\mathrm{x}_{i}}^{\mathrm{x}} F^{\prime}(t) d t=\left[2 F^{\prime}\left(\frac{3 \mathrm{x}_{i}+\mathrm{x}}{4}\right)-F^{\prime}\left(\frac{\mathrm{x}_{i}+\mathrm{x}}{2}\right)+2 F^{\prime}\left(\frac{\mathrm{x}_{i}+3 \mathrm{x}}{4}\right)\right] \frac{\left(\mathrm{x}-\mathrm{x}_{i}\right)}{3}
$$


From (3) and (9), we have,

$$
F(\mathrm{x})=F\left(\mathrm{x}_{i}\right)+\left[2 F^{\prime}\left(\frac{3 \mathrm{x}_{i}+\mathrm{x}}{4}\right)-F^{\prime}\left(\frac{\mathrm{x}_{i}+\mathrm{x}}{2}\right)+2 F^{\prime}\left(\frac{\mathrm{x}_{i}+3 \mathrm{x}}{4}\right)\right] \frac{\left(\mathrm{x}-\mathrm{x}_{i}\right)}{3}
$$

Since, $F(\mathrm{x})=0$ then

$$
\mathrm{x}=\mathrm{x}_{i}-3\left[2 F^{\prime}\left(\frac{3 \mathrm{x}_{i}+\mathrm{x}}{4}\right)-F^{\prime}\left(\frac{\mathrm{x}_{i}+\mathrm{x}}{2}\right)+2 F^{\prime}\left(\frac{\mathrm{x}_{i}+3 \mathrm{x}}{4}\right)\right]^{-1} F\left(\mathrm{x}_{i}\right)
$$

which is an implicit method to compute the approximate solution implicitly, we use the prediction and correction technique. With the formulation (11) for solving (SNLE) (2) as follows:

Algorithm 2: For a given $\mathrm{X}_{0}$, compute the approximate solution $\mathrm{X}_{i+1}$ by iterative scheme.

$$
\begin{aligned}
& \mathrm{y}_{i}=\mathrm{x}_{i}-J^{-1}\left(\mathrm{x}_{i}\right) F\left(\mathrm{x}_{i}\right), \\
& \mathrm{x}_{\mathrm{i}+1}=\mathrm{x}_{i}-3\left[2 F^{\prime}\left(\frac{3 \mathrm{x}_{i}+\mathrm{y}_{\mathrm{i}}}{4}\right)-F^{\prime}\left(\frac{\mathrm{x}_{i}+\mathrm{y}_{\mathrm{i}}}{2}\right)+2 F^{\prime}\left(\frac{\mathrm{x}_{i}+3 \mathrm{y}_{\mathrm{i}}}{4}\right)\right]^{-1} F\left(\mathrm{x}_{i}\right)
\end{aligned}
$$

Furthermore, if we approximate $\int_{x_{i}}^{x} F^{\prime}(t) d t$ by using two point Newton-Cotes, Open formula [27], then we have

$$
\int_{\mathrm{x}_{i}}^{\mathrm{x}} F^{\prime}(t) d t=\left[F^{\prime}\left(\frac{2 \mathrm{x}_{i}+x}{3}\right)+F^{\prime}\left(\frac{\mathrm{x}_{i}+2 x}{3}\right)\right] \frac{\left(\mathrm{x}-\mathrm{x}_{i}\right)}{2}
$$

From (3) and (13), we have

$$
F(\mathrm{x})=F\left(\mathrm{x}_{i}\right)+\left[F^{\prime}\left(\frac{2 \mathrm{x}_{i}+x}{3}\right)+F^{\prime}\left(\frac{\mathrm{x}_{i}+2 x}{3}\right)\right] \frac{\left(\mathrm{x}-\mathrm{x}_{i}\right)}{2}
$$

Since, $F(\mathrm{x})=0$ then

$$
\mathrm{x}_{i+1}=\mathrm{x}_{i}-2\left[F^{\prime}\left(\frac{2 \mathrm{x}_{i}+x}{3}\right)+F^{\prime}\left(\frac{\mathrm{x}_{i}+2 x}{3}\right)\right]^{-1} F\left(\mathrm{x}_{i}\right)
$$

to compute the approximate solution implicitly, we use the prediction and correction technique. With the formulation (15) for solving the system of nonlinear equation (2) as follows:

Algorithm 3: For a given $\mathrm{X}_{0}$, compute the approximate solution $\mathrm{X}_{i+1}$ by iterative scheme

$$
\begin{aligned}
& \mathrm{y}_{i}=\mathrm{x}_{i}-J^{-1}\left(\mathrm{x}_{i}\right) F\left(\mathrm{x}_{i}\right), \\
& \mathrm{x}_{i+1}=\mathrm{x}_{i}-2\left[F^{\prime}\left(\frac{2 \mathrm{x}_{i}+y_{i}}{3}\right)+F^{\prime}\left(\frac{\mathrm{x}_{i}+2 y_{i}}{3}\right)\right]^{-1} F\left(\mathrm{x}_{i}\right)
\end{aligned}
$$

Finally, if we approximate $\int_{x_{i}}^{x} F^{\prime}(t) d t$ using four points Newton-Cotes, Closed formula [27], then we have

$$
\int_{\mathrm{x}_{i}}^{\mathrm{x}} F^{\prime}(t) d t=\left[F^{\prime}\left(\mathrm{x}_{i}\right)+3 F^{\prime}\left(\frac{2 \mathrm{x}_{i}+x}{3}\right)+3 F^{\prime}\left(\frac{\mathrm{x}_{i}+2 x}{3}\right)+F^{\prime}(\mathrm{x})\right] \frac{\left(\mathrm{x}-\mathrm{x}_{i}\right)}{8}
$$


From (3) and (18), we have

$$
F(\mathrm{x})=F\left(\mathrm{x}_{i}\right)+\left[F^{\prime}\left(\mathrm{x}_{i}\right)+3 F^{\prime}\left(\frac{2 \mathrm{x}_{i}+x}{3}\right)+3 F^{\prime}\left(\frac{\mathrm{x}_{i}+2 x}{3}\right)+F^{\prime}(\mathrm{x})\right] \frac{\left(\mathrm{x}-\mathrm{x}_{i}\right)}{8}
$$

Since, $F(\mathrm{x})=0$ then

$$
\mathrm{x}_{i+1}=\mathrm{x}_{i}-8\left[F^{\prime}\left(\mathrm{x}_{i}\right)+3 F^{\prime}\left(\frac{2 \mathrm{x}_{i}+x}{3}\right)+3 F^{\prime}\left(\frac{\mathrm{x}_{i}+2 x}{3}\right)+F^{\prime}(x)\right]^{-1} F\left(\mathrm{x}_{i}\right)
$$

by using the prediction and correction technique. With the formulation (20) the system of nonlinear equation (2) as follows:

Algorithm 4: For a given $\mathrm{X}_{0}$, compute the approximate solution $\mathrm{X}_{i+1}$ by iterative scheme.

$$
\begin{aligned}
& \mathrm{y}_{i}=\mathrm{x}_{i}-J^{-1}\left(\mathrm{x}_{i}\right) F\left(\mathrm{x}_{i}\right), \\
& \mathrm{x}_{i+1}=\mathrm{x}_{i}-8\left[F^{\prime}\left(\mathrm{x}_{i}\right)+3 F^{\prime}\left(\frac{2 \mathrm{x}_{i}+y_{i}}{3}\right)+3 F^{\prime}\left(\frac{\mathrm{x}_{i}+2 y_{i}}{3}\right)+F^{\prime}\left(y_{i}\right)\right]^{-1} F\left(\mathrm{x}_{i}\right)
\end{aligned}
$$

\section{Convergence analysis}

In this section, we consider the convergence of our algorithm using the Taylor's series technique.

Theorem 1: Let $\mathrm{x}^{*}$ be a sample zero of sufficient differentiable function $F: \subseteq R^{n} \rightarrow R^{n}$ for an open interval. If $0 \mathrm{x}$ is sufficiently close to $\mathrm{x}^{*}$, then the two step method defined by our algorithm (5)has convergence is at least of order 3.

Proof. Consider to

$$
\begin{aligned}
& \mathrm{y}_{n}=\mathrm{x}_{\mathrm{n}}-\frac{F\left(\mathrm{x}_{\mathrm{n}}\right)}{F^{\prime}\left(\mathrm{x}_{\mathrm{n}}\right)}, \\
& \mathrm{x}_{\mathrm{n}+1}=\mathrm{x}_{\mathrm{n}}-6\left[F^{\prime}\left(\mathrm{x}_{\mathrm{n}}\right)+4 F\left(\mathrm{w}_{\mathrm{n}}\right)+F^{\prime}\left(\mathrm{y}_{\mathrm{n}}\right)\right]^{-1} F\left(\mathrm{x}_{\mathrm{n}}\right)
\end{aligned}
$$

where $\mathrm{w}_{n}=\frac{1}{2}\left(\mathrm{x}_{n}+\mathrm{y}_{n}\right)$, Let $\mathrm{x}^{*}$ be a sample zero of $F$. Since $F$ is sufficiently differentiable, by expanding $F\left(\mathrm{x}_{n}\right)$ and $F^{\prime}\left(\mathrm{x}_{n}\right)$ about $\mathrm{x}^{*}$, we get

$$
F\left(\mathrm{x}_{n}\right)=F\left(\mathrm{x}^{*}\right)+F^{\prime}\left(\mathrm{x}^{*}\right)\left(\mathrm{x}_{n}-\mathrm{x}^{*}\right)+F^{(2)}\left(\mathrm{x}^{*}\right) \frac{\left(\mathrm{x}_{n}-\mathrm{x}^{*}\right)^{2}}{2 !}+F^{(3)}\left(\mathrm{x}^{*}\right) \frac{\left(\mathrm{x}_{n}-\mathrm{x}^{*}\right)^{3}}{3 !}+\cdots,
$$

But $F\left(\mathrm{x}^{*}\right)=0$ then

$$
F\left(\mathrm{x}_{n}\right)=F^{\prime}\left(\mathrm{x}^{*}\right)\left[E_{n}+C_{2} E_{n}^{2}+C_{3} E_{n}^{3}+O\left(\left\|E_{n}^{4}\right\|\right)\right]
$$

and

$$
F^{\prime}\left(\mathrm{x}_{n}\right)=F^{\prime}\left(\mathrm{x}^{*}\right)\left[I+2 C_{2} E_{n}+3 C_{3} E_{n}^{2}+O\left(\left\|E_{n}^{3}\right\|\right)\right],
$$

where $C_{k}=\frac{1}{k !} \frac{F^{(k)}\left(\mathrm{x}^{*}\right)}{F^{\prime}\left(\mathrm{x}^{*}\right)}, k=2,3, \ldots$ and $E_{n}=\mathrm{x}_{n}-\mathrm{x}^{*}$.

Now from (25) and (26), we have (see [28])

$$
\frac{F\left(\mathrm{x}_{n}\right)}{F^{\prime}\left(\mathrm{x}_{n}\right)}=E_{n}-C_{2} E_{n}^{2}+2\left(C_{2}^{2}-C_{3}\right) E_{n}^{3}+O\left(\left\|E_{n}^{4}\right\|\right),
$$

From (23) and (27), we get

$$
\mathrm{y}_{n}=\mathrm{x}^{*}+C_{2} E_{n}^{2}+2\left(C_{3}-C_{2}^{2}\right) E_{n}^{3}+O\left(\left\|E_{n}^{4}\right\|\right),
$$


From (22), we get,

$$
F\left(\mathrm{y}_{n}\right)=F^{\prime}\left(\mathrm{x}^{*}\right)\left[\left(\mathrm{y}_{n}-\mathrm{x}^{*}\right)+C_{2}\left(\mathrm{y}_{n}-\mathrm{x}^{*}\right)^{2}+C_{3}\left(\mathrm{y}_{n}-\mathrm{x}^{*}\right)^{3}+O\left(\left\|E_{n}^{4}\right\|\right)\right]
$$

and

$$
\begin{aligned}
F^{\prime}\left(\mathrm{y}_{n}\right) & =F^{\prime}\left(\mathrm{x}^{*}\right)\left[I+2 C_{2}\left(\mathrm{y}_{n}-\mathrm{x}^{*}\right)+3 C_{3}\left(\mathrm{y}_{n}-\mathrm{x}^{*}\right)^{2}+O\left(\left\|E_{n}^{3}\right\|\right)\right] \\
& =F^{\prime}\left(\mathrm{x}^{*}\right)\left[I+2 C_{2}^{2} E_{n}^{2}+O\left(\left\|E_{n}^{3}\right\|\right)\right] .
\end{aligned}
$$

Expanding $F^{\prime}\left(\mathrm{w}_{n}\right)$ about $\mathrm{x}^{*}$, we get

$$
F^{\prime}\left(\mathrm{w}_{n}\right)=F^{\prime}\left(\mathrm{x}^{*}\right)\left[I+2 C_{2}\left(\mathrm{w}_{n}-\mathrm{x}^{*}\right)+3 C_{3}\left(\mathrm{w}_{n}-\mathrm{x}^{*}\right)^{2}+O\left(\left\|E_{n}^{3}\right\|\right)\right]
$$

Where $\mathrm{w}_{n}=\frac{1}{2}\left(\mathrm{x}_{n}+\mathrm{y}_{n}\right)$ from (22) we have

then

$$
F^{\prime}\left(\mathrm{w}_{n}\right)=F^{\prime}\left(\mathrm{x}^{*}\right)\left[I+C_{2} E_{n}+\left(C_{2}^{2}+\frac{3}{4} C_{3}\right) E_{n}^{2}+O\left(\left\|E_{n}^{3}\right\|\right)\right]
$$

$$
\begin{aligned}
F^{\prime}\left(\mathrm{x}_{n}\right)+4 F^{\prime}\left(\mathrm{w}_{n}\right)+F^{\prime}\left(\mathrm{y}_{n}\right) & =F^{\prime}\left(\mathrm{x}^{*}\right)\left[6 I+6 C_{2} E_{n}+\left(6 C_{2}^{2}+9 C_{3}\right) E_{n}^{2}+O\left(\left\|E_{n}^{3}\right\|\right)\right] \\
& =6 F^{\prime}\left(\mathrm{x}^{*}\right)\left[I+C_{2} E_{n}+\left(C_{2}^{2}+\frac{3}{2} C_{3}\right) E_{n}^{2}+O\left(\left\|E_{n}^{3}\right\|\right)\right]
\end{aligned}
$$

From (24), $E_{n+1}=\mathrm{x}_{n+1}-\mathrm{x}^{*}$ and $E_{n}=\mathrm{x}_{n}-\mathrm{x}^{*}$.

$$
\begin{aligned}
& E_{n+1}=E_{n}-6\left[J\left(\mathrm{x}_{n}\right)+4 J\left(\mathrm{w}_{n}\right)+J\left(\mathrm{y}_{n}\right)\right]^{-1} F\left(\mathrm{x}_{n}\right) \\
& E_{n+1}=E_{n}-6\left[6 F^{\prime}\left(\mathrm{x}^{*}\right)\left[I+C_{2} E_{n}+\left(C_{2}^{2}+\frac{3}{2} C_{3}\right) E_{n}^{2}+O\left(\left\|E_{n}^{3}\right\|\right)\right]\right]^{-1} F\left(\mathrm{x}_{n}\right)
\end{aligned}
$$

In general, according to [28]:

$$
\left[I+M_{2} E_{n}+M_{3} E_{n}^{2}+O_{3}\right]^{-1}=\left[I-M_{2} E_{n}+\left(M_{2}^{2}-M_{3}\right) E_{n}^{2}+O_{3}\right]
$$

Set $M_{2}=C_{2}, M_{3}=C_{2}^{2}+\frac{3}{2} C_{3}$

Then we will have

$$
E_{n+1}=E_{n}-\left\{F^{\prime}\left(\mathrm{x}^{*}\right)\left[I-C_{2} E_{n}-\frac{3}{2} C_{3} E_{n}^{2}+O\left(\left\|E_{n}^{3}\right\|\right)\right]\right\} F\left(\mathrm{x}_{n}\right)
$$

From (25)

$$
E_{n+1}=E_{n}-\left[I-C_{2} E_{n}-\frac{3}{2} C_{3} E_{n}^{2}+O\left(\left\|E_{n}^{3}\right\|\right)\right]\left[E_{n}+C_{2} E_{n}^{2}+C_{3} E_{n}^{3}+O\left(\left\|E_{n}^{4}\right\|\right)\right]
$$

Finally,

$$
E_{n+1}=\left(C_{2}^{2}+\frac{1}{2} C_{3}\right) E_{n}^{3}+O\left(\left\|E_{n}^{4}\right\|\right)
$$

which shows that Algorithm (1) is at least a third order convergent method, the required result. Since asymptotic convergence of Newton method is $2 \mathrm{c}$ and from Theorem 1, we deduce that the convergence rate of our algorithm is better than the Newton's method. And the cubic convergent method is vastly superior to the linear and the quadratically convergent methods [29]. 


\section{Numerical examples}

For comparisons, we have used the third-order Hafiz and Bahgat method (HBM) [16] and Darvishi (DAM) [14] algorithms, respectively.

$$
\begin{aligned}
& \mathrm{x}_{n+1}=\mathrm{x}_{n}-12\left[J\left(\mathrm{x}_{n}\right)+10 J\left(\mathrm{w}_{n}\right)+J\left(\mathrm{y}_{n}\right)\right]^{-1} F\left(\mathrm{x}_{n}\right), \\
& \mathrm{x}_{n+1}=\mathrm{x}_{n}-2\left[F^{\prime}\left(\mathrm{x}_{n}\right)+F^{\prime}\left(\mathrm{y}_{n}\right)\right]^{-1} F\left(\mathrm{x}_{n}\right),
\end{aligned}
$$

where $\mathrm{y}_{n}=\mathrm{x}_{n}-J^{1}\left(\mathrm{x}_{n}\right) F\left(\mathrm{x}_{n}\right)$.

We present some examples to illustrate the efficiency of our proposed methods. Here, numerical results are performed by Maple 15 with 200 digits but only 14 digits are displayed. In Tables [1-5] we list the results obtained in Algorithm [1-4], which we called, Khirallah and Hafiz Methods (KHM1, KHM2, KHM3, KHM4), respectively and compare them with Newton-Raphson method (NM), Hafiz and Bahgat method (HBM) [16], and Darvishi (DAM). The following stopping criteria is used for computer programs:

$$
\left\|\mathrm{x}^{(n+1)}-\mathrm{x}^{(n)}\right\|+\left\|F\left(\mathrm{x}^{(n)}\right)\right\|<10^{-15}
$$

and the computational order of convergence (COC) can be approximated using the following formula:

$$
\mathrm{COC} \approx \frac{\ln \left(\left\|\mathrm{x}^{(n+1)}-\mathrm{x}^{(n)}\right\| /\left\|\mathrm{x}^{(n)}-\mathrm{x}^{(n-1)}\right\|\right)}{\ln \left(\left\|\mathrm{x}^{(n)}-\mathrm{x}^{(n-1)}\right\| /\left\|\mathrm{x}^{(n-1)}-\mathrm{x}^{(n-2)}\right\|\right)}
$$

Table 2 shows the number of iterations and the computational order of convergence (COC). $\left\|\mathrm{x}^{(n+1)}-\mathrm{x}^{(n)}\right\|$ and the norm of the function $F\left(\mathrm{x}^{(n)}\right)$ is also shown in Table 2 for various methods.

Table 1. Number of iterations for Example 1.

\begin{tabular}{|l|l|l|l|l|}
\hline $\begin{array}{l}\text { Functions \& } \\
\text { Methods }\end{array}$ & IT & \multicolumn{1}{|c|}{ COC } & $\left\|\mathrm{x}^{(n+1)}-\mathrm{x}^{(n)}\right\|_{2}$ & $\left\|{ }^{2}\left(\mathrm{x}^{(n)}\right)\right\|_{2}$ \\
\hline$F_{1}, \mathrm{x}_{0}=-3$. & & & & \\
\hline NM & 15 & 2.00000001842997 & $4.62461580585008 \mathrm{E}-28$ & $6.52399684550115 \mathrm{E}-54$ \\
\hline HBM & 10 & 2.99999933808324 & $1.05721596802914 \mathrm{E}-38$ & $4.81394938579303 \mathrm{E}-113$ \\
\hline DAM & 10 & 2.99922897924987 & $3.70578223790695 \mathrm{E}-18$ & $3.36653193192208 \mathrm{E}-51$ \\
\hline KHM1 & 10 & 2.99922897924987 & $3.70578223790695 \mathrm{E}-18$ & $3.36653193192208 \mathrm{E}-51$ \\
\hline KHM2 & 10 & 2.99999480789091 & $3.01303999741227 \mathrm{E}-33$ & $1.2533863797071 \mathrm{E}-96$ \\
\hline KHM3 & 10 & 2.99999974385679 & $6.65989967725116 \mathrm{E}-41$ & $1.15336367836786 \mathrm{E}-119$ \\
\hline KHM4 & 10 & 2.99999461902748 & $4.01650938788201 \mathrm{E}-33$ & $2.96904403993932 \mathrm{E}-96$ \\
\hline$F_{2}, \mathrm{x}_{0}=4$ & & & & \\
\hline NM & 20 & 2.00000177738031 & $7.04037622176027 \mathrm{E}-21$ & $4.23796972290579 \mathrm{E}-39$ \\
\hline HBM & 14 & 2.99983859733573 & $1.59297520186683 \mathrm{E}-25$ & $2.08149877265886 \mathrm{E}-72$ \\
\hline DAM & 14 & 2.99980584903215 & $1.86433543978608 \mathrm{E}-25$ & $4.87234659996821 \mathrm{E}-72$ \\
\hline KHM1 & 13 & 2.99927894804511 & $9.88081115604762 \mathrm{E}-21$ & $5.42458694342177 \mathrm{E}-58$ \\
\hline KHM2 & 13 & 2.99930488585053 & $7.47548441892867 \mathrm{E}-21$ & $2.34912917724958 \mathrm{E}-58$ \\
\hline KHM3 & 13 & 2.99991089189577 & $1.8889427520925 \mathrm{E}-27$ & $3.36412572863912 \mathrm{E}-78$ \\
\hline KHM4 & 13 & 2.99928670711787 & $9.09952966462796 \mathrm{E}-21$ & $4.23687559822413 \mathrm{E}-58$ \\
\hline
\end{tabular}




\subsection{Small systems of nonlinear equations}

Example 1. In a case of one dimension, consider the following nonlinear functions [12], $f_{1}(x)=x e^{x^{2}}-\sin ^{2} x+3 \cos x+5$, with $x_{0}=-3$ and $f_{2}(x)=e^{x^{2}+7 x-30}-1$ with $x_{0}=4$.

Example 2. In a case two dimension, consider the following systems of nonlinear functions [30], $F_{3}(\mathrm{x})=\left\{\begin{array}{l}f_{1}(x, y)=x^{2}-10 x+y^{2}+8=0 \\ f_{2}(x, y)=x y^{2}+x-10 y+8=0\end{array},\left(x_{0}, y_{0}\right)=(2,2)\right.$. $F_{4}(\mathrm{x})=\left\{\begin{array}{l}f_{1}(x, y)=x^{4} y-x y+2 x-y-1=0 \\ f_{2}(x, y)=y e^{-x}+x-y-e^{-1}=0\end{array},\left(x_{0}, y_{0}\right)=(0.8,0.8)\right.$.

Example 3. In a case three dimension, consider the following systems of nonlinear functions [30].

$$
\begin{aligned}
& F_{5}(\mathrm{x})=\left\{\begin{array}{l}
f_{1}(x, y, z)=15 x+y^{2}-4 z-13=0 \\
f_{2}(x, y, z)=x^{2}+10 y-e^{-z}-11=0, X_{0}=(10,6,-5) . \\
f_{3}(x, y, z)=y^{3}-25 z+22=0
\end{array}\right. \\
& F_{6}(\mathrm{x})=\left\{\begin{array}{l}
f_{1}(x, y, z)=3 x-\cos (y z)-0.5=0 \\
f_{2}(x, y, z)=x^{2}-81(y+0.1)^{2}+\sin z+1.06=0 \\
f_{3}(x, y, z)=e^{-x y}+20 z+\frac{10 \pi-3}{3}=0
\end{array}, X_{0}=(1.1,1.1,1.1) .\right.
\end{aligned}
$$

Table 2. Number of iterations for Example 2.

\begin{tabular}{|l|l|l|l|l|}
\hline $\begin{array}{l}\text { Methods \& } \\
\text { functions }\end{array}$ & IT & \multicolumn{1}{|c|}{ COC } & $\left\|\mathrm{x}^{(n+1)}-\mathrm{x}^{(n)}\right\|_{2}$ & \multicolumn{1}{|c|}{$\left\|F\left(\mathrm{x}^{(n)}\right)\right\|_{2}$} \\
\hline$F_{3}, \mathrm{X}_{0}=(2,2)$ & & & & \\
\hline NM & 9 & 2.00000140914961 & $4.37934644418127 \mathrm{E}-21$ & $8.47815841458272 \mathrm{E}-41$ \\
\hline HBM & 6 & 3.00155165046022 & $2.89152305962132 \mathrm{E}-20$ & $5.42470874639365 \mathrm{E}-59$ \\
\hline DAM & 6 & 3.00384615129491 & $2.35456290038799 \mathrm{E}-17$ & $3.23994953735791 \mathrm{E}-50$ \\
\hline KHM1 & 6 & 3.0018969782973 & $1.28314015297702 \mathrm{E}-19$ & $4.8413637367635 \mathrm{E}-57$ \\
\hline KHM2 & 6 & 3.0018969782973 & $1.28314015297702 \mathrm{E}-19$ & $4.8413637367635 \mathrm{E}-57$ \\
\hline KHM3 & 6 & 3.00144738591666 & $1.7256860796277 \mathrm{E}-20$ & $1.14494920674716 \mathrm{E}-59$ \\
\hline KHM4 & 6 & 3.0018969782973 & $1.28314015297702 \mathrm{E}-19$ & $4.8413637367635 \mathrm{E}-57$ \\
\hline$F_{4}, \mathrm{X}_{0}=(2,2)$ & & & & \\
\hline NM & 9 & 2.0003080790074 & $7.53061926402066 \mathrm{E}-19$ & $2.30110082351703 \mathrm{E}-36$ \\
\hline HBM & 6 & 3.00233622614329 & $1.18980970698501 \mathrm{E}-18$ & $1.02194895526103 \mathrm{E}-53$ \\
\hline DAM & 7 & 3.00021436384324 & $1.17783537421347 \mathrm{E}-43$ & $1.37875704566421 \mathrm{E}-128$ \\
\hline KHM1 & 6 & 3.00174818657116 & $7.02465997001949 \mathrm{E}-18$ & $2.26756512830416 \mathrm{E}-51$ \\
\hline KHM2 & 6 & 3.00174798833245 & $7.00487395265092 \mathrm{E}-18$ & $2.24845784528931 \mathrm{E}-51$ \\
\hline KHM3 & 6 & 3.00251772607513 & $6.41769417427871 \mathrm{E}-19$ & $1.56198405876415 \mathrm{E}-54$ \\
\hline KHM4 & 6 & 3.00174812784528 & $7.01879180165014 \mathrm{E}-18$ & $2.26188701404281 \mathrm{E}-51$ \\
\hline
\end{tabular}


Table 3. Number of iterations for Example 3.

\begin{tabular}{|l|l|l|l|l|}
\hline $\begin{array}{l}\text { Methods \& } \\
\text { functions }\end{array}$ & IT & \multicolumn{1}{|c|}{ COC } & $\left\|\mathrm{x}^{(n+1)}-\mathrm{x}^{(n)}\right\|_{2}$ & $\left\|F\left(\mathrm{x}^{(n)}\right)\right\|_{2}$ \\
\hline$F_{5}, \mathrm{X}_{0}=(10,6,-5)$ & & & & \\
\hline NM & 8 & 2.26089072336615 & $1.30827439612494 \mathrm{E}-16$ & $5.41580264395725 \mathrm{E}-32$ \\
\hline HBM & 5 & 3.11050550213485 & $6.09915403468275 \mathrm{E}-22$ & $2.91039586070281 \mathrm{E}-65$ \\
\hline ADM & 6 & 3.19324326204977 & $5.23998126509222 \mathrm{E}-19$ & $6.94886127056805 \mathrm{E}-56$ \\
\hline KHM1 & 5 & 3.23147391984341 & $1.81427047173709 \mathrm{E}-16$ & $1.18314011923307 \mathrm{E}-49$ \\
\hline KHM2 & 5 & 3.22620879334894 & $4.10586956837708 \mathrm{E}-18$ & $1.11920543355639 \mathrm{E}-54$ \\
\hline KHM3 & 5 & 3.24321465344843 & $8.23705630178016 \mathrm{E}-17$ & $6.49793389946669 \mathrm{E}-50$ \\
\hline KHM4 & 5 & 3.22914844338236 & $7.05132295496965 \mathrm{E}-17$ & $6.82403435648476 \mathrm{E}-51$ \\
\hline$F_{6}, X_{0}=(1.1,1.1,1.1)$ & & & & \\
\hline NM & 9 & 2.0000000032895 & $6.45188221156175 \mathrm{E}-20$ & $3.36918186538804 \mathrm{E}-37$ \\
\hline HBM & 6 & 2.99634279195962 & $4.93648808913231 \mathrm{E}-19$ & $4.87596025092796 \mathrm{E}-53$ \\
\hline DAM & 6 & 2.99606536543964 & $8.48219679389847 \mathrm{E}-19$ & $2.4736197503045 \mathrm{E}-52$ \\
\hline KHM1 & 6 & 2.99627938972062 & $5.60696523234285 \mathrm{E}-19$ & $7.14479665255229 \mathrm{E}-53$ \\
\hline KHM2 & 6 & 2.99626324434839 & $5.78960142628486 \mathrm{E}-19$ & $7.86596995617356 \mathrm{E}-53$ \\
\hline KHM3 & 6 & 2.99635743716414 & $4.79178354244989 \mathrm{E}-19$ & $4.45961446054831 \mathrm{E}-53$ \\
\hline KHM4 & 6 & 2.99627454598102 & $5.66122916703125 \mathrm{E}-19$ & $7.35425170801941 \mathrm{E}-53$ \\
\hline
\end{tabular}

\subsection{Large systems of nonlinear equations}

In this subsection, we test HPM with some sparse systems with m unknown variables. In examples [4-6], we compare the NR method with the proposed HPM method focusing on iteration numbers $[15]$.

Table 4. Number of iterations for Examples 4-6.

\begin{tabular}{|l|c|c|c|c|c|c|c|c|c|}
\hline Methods & $F 7$ & $F 8$ & $F 9$ & $F 7$ & $F 8$ & $F 9$ & $F 7$ & $F 8$ & $F 9$ \\
\hline$\varepsilon=10^{-15}$ & \multicolumn{3}{|c|}{$m=50$} & \multicolumn{3}{|c|}{$m=75$} & \multicolumn{3}{|c|}{$m=100$} \\
\hline NM & 6 & 7 & 53 & 6 & 7 & 53 & 6 & 7 & 53 \\
\hline HBM & 4 & 5 & 34 & 4 & 5 & 34 & 4 & 5 & 35 \\
\hline DAM & 5 & 5 & 33 & 5 & 5 & 34 & 5 & 5 & 34 \\
\hline KHM1 & 4 & 5 & 34 & 4 & 5 & 35 & 4 & 5 & 35 \\
\hline KHM2 & 4 & 5 & 34 & 4 & 5 & 35 & 4 & 5 & 35 \\
\hline KHM3 & 4 & 5 & 35 & 4 & 5 & 35 & 4 & 5 & 35 \\
\hline KHM4 & 4 & 5 & 34 & 4 & 5 & 35 & 4 & 5 & 35 \\
\hline
\end{tabular}


Example 4. Consider the following system of nonlinear equations:

$$
F_{7}: f_{i}=e^{x_{i}}-1, \quad i=1,2, \ldots, m \text {. }
$$

The exact solution of this system is $X^{*}=[0,0, \ldots, 0]^{T}$. To solve this system, we set $\mathrm{x}_{0}[0.5,0.5, \ldots, 0.5]^{T}$ as an initial value. Tables 4,5 are shown the result.

Example 5. Consider the following system of nonlinear equations:

$$
F_{8}: f_{i}=x_{i}^{2}-\cos \left(x_{i}-1\right), \quad i=1,2, \ldots, m .
$$

One of the exact solutions of this system is $X^{*}=[1,1, \ldots, 1]^{T}$. To solve this system, we set $\mathrm{x}_{0}=[2,2 ., \ldots, 2]^{\mathrm{T}}$ as an initial value. The results are presented in Tables 4, 5 .

Example 6. Consider the following system of nonlinear equations [13]:

$$
F_{9}: f_{i}=\cos x_{i}-1, \quad i=1,2, \ldots, m .
$$

One of the exact solutions of this system is $x^{*}=[0,0, \ldots, 0]^{T}$. To solve this system, we set $\mathrm{x}_{0}=[2,2, \ldots, 2]^{\mathrm{T}}$ as an initial guess. The results are presented in Tables 4, 5 .

Example 7. Consider the nonlinear boundary value problem [17]

$$
y^{\prime \prime}=-\left(y^{\prime}\right)^{2}-y+\ln x, 1 \leq x \leq 2, y(1)=0, y(2)=\ln 2 .
$$

whose exact solution is $\mathrm{y}=\ln \mathrm{x}$. We consider the following partition of the interval:

$$
x_{0}=1, x_{n}=2, x_{j}=x_{0}+j h, h=\frac{1}{m}, j=1,2, \cdots, m-1
$$

Let us define now

$$
y_{0}=y\left(x_{0}\right)=0, y_{m}=\ln 2, y_{i}=f\left(x_{i}\right), i=1,2, \cdots, m-1 .
$$

If we discretize the problem by using the second order finite differences method defined by the numerical formulas

$$
\begin{aligned}
& y_{i}^{\prime}=\frac{y_{i+1}-y_{i-1}}{2 h}, i=1,2, \cdots, m-1, \\
& y_{i}^{\prime \prime}=\frac{y_{i+1}-2 y_{i}+y_{i-1}}{h^{2}}, i=1,2, \cdots, m-1,
\end{aligned}
$$

then, we obtain a $(\mathrm{m}-1) \times(\mathrm{m}-1)$ system of nonlinear equations $F_{10}$ :

$$
\begin{aligned}
& 4 y_{2}+y_{2}^{2}+4 y_{1}\left(h^{2}-2\right)-4 h^{2} \ln x_{2}=0, \\
& 4\left(y_{i+1}+y_{i-1}\right)+\left(y_{i+1}-y_{i-1}\right)^{2}+4 y_{i}\left(h^{2}-2\right)-4 h^{2} \ln x_{i+1}=0, \quad i=2, \cdots, m-1, \\
& 4\left(\ln 2+y_{m-2}\right)+\left(\ln 2-y_{m-2}\right)^{2}+4 y_{m-1}\left(h^{2}-2\right)-4 h^{2} \ln x_{m}=0
\end{aligned}
$$

We take $\mathrm{X}_{0}$ with $y_{k}^{(0)}=\ln \left(\frac{k}{2}\right), k=1,2, \cdots, m-1$, as a starting point. In particular, we solve this problem for $\mathrm{m}=50,75$ and 100 . The numerical results for the above system of nonlinear equations are presented in Table 5. The number of iterations of methods HBM, KHM1, KHM2, KHM3 and KHM4 are equal but method KHM2, ... KHM4 has advantage of they are free from second derivatives, over methods NM and HBM, because the cost of computing second derivatives is very high, see Table 6 . 
Table 5. Comparison of the computational order of convergence (COC) for Examples 4-6.

\begin{tabular}{|c|c|c|c|c|c|c|c|c|c|}
\hline Methods & $F 7$ & $F 8$ & F9 & $F 7$ & $F 8$ & F9 & $F 7$ & $F 8$ & F9 \\
\hline$\varepsilon=10^{-15}$ & \multicolumn{3}{|c|}{$m=50$} & \multicolumn{3}{|c|}{$m=75$} & \multicolumn{3}{|c|}{$m=100$} \\
\hline NM & 2.00 & 1.99 & 1.00 & 2.00 & 1.99 & 1.00 & 2.00 & 1.99 & 1.00 \\
\hline HBM & 2.99 & 2.99 & 1.00 & 2.99 & 2.99 & 1.00 & 2.99 & 2.99 & 1.00 \\
\hline DAM & 2.99 & 2.99 & 1.00 & 2.99 & 2.99 & 1.00 & 2.99 & 2.99 & 1.00 \\
\hline KHM1 & 2.99 & 2.99 & 1.00 & 2.99 & 2.99 & 1.00 & 2.99 & 2.99 & 1.00 \\
\hline KHM2 & 2.99 & 2.99 & 1.00 & 2.99 & 2.99 & 1.00 & 2.99 & 2.99 & 1.00 \\
\hline KHM3 & 2.99 & 2.99 & 1.00 & 2.99 & 2.99 & 1.00 & 2.99 & 2.99 & 1.00 \\
\hline KHM4 & 2.99 & 2.99 & 1.00 & 2.99 & 2.99 & 1.00 & 2.99 & 2.99 & 1.00 \\
\hline
\end{tabular}

In Tables 1-6, we list the results obtained by modified iterations methods. As we see from this Tables, it is clear that, in most cases, the result obtained by DAM, HBM, KHM1,... KHM4 are equivalent and they very superior to that obtained NM.

\section{Conclusions}

In this paper, we presented four new algorithms for solving the system of nonlinear equations by using Simpson's formula, and Newton's two, three and four Cosed formulas iterative methods and used these algorithms for the first time for solving initial value problem. These methods have the same efficiency as the other third-order methods in the literature. We conclude from the numerical examples that the proposed methods have at least equal performance as compared with the other methods of the same order. Moreover, our proposed methods provid

Table 6. Number of iterations for Examples 7.

\begin{tabular}{|l|c|c|c|c|c|}
\hline \multicolumn{1}{|c|}{ Methods } & $m$ & $\mathrm{IT}$ & \multicolumn{1}{c|}{ COC } & $\left\|\mathrm{x}^{(n+1)}-\mathrm{x}^{(n)}\right\|_{2}$ & $\left\|F\left(\mathrm{x}^{(n)}\right)\right\|_{2}$ \\
\hline F10 & & & & & \\
\hline NM & & 9 & 1.99590511842648 & $5.55653355591706 \mathrm{E}-22$ & $3.36918186538804 \mathrm{E}-37$ \\
\hline HBM & & 6 & 2.95997350151316 & $7.30961049290975 \mathrm{E}-43$ & $1.85852506647941 \mathrm{E}-128$ \\
\hline DAM & & 6 & 2.95997350151316 & $7.30961049290975 \mathrm{E}-43$ & $1.85852506647941 \mathrm{E}-128$ \\
\hline KHM1 & 50 & 6 & 2.95997350151316 & $7.30961049290975 \mathrm{E}-43$ & $1.85852506647941 \mathrm{E}-128$ \\
\hline KHM2 & & 6 & 2.95997350151316 & $7.30961049290975 \mathrm{E}-43$ & $1.85852506647941 \mathrm{E}-128$ \\
\hline KHM3 & & 6 & 2.95997350151316 & $7.30961049290975 \mathrm{E}-43$ & $1.85852506647941 \mathrm{E}-128$ \\
\hline KHM4 & & 6 & 2.95997350151316 & $7.30961049290975 \mathrm{E}-43$ & $1.85852506647941 \mathrm{E}-128$ \\
\hline$F_{10}$ & & & & & \\
\hline NM & & 7 & 2.00919187729643 & $6.66382516768248 \mathrm{E}-20$ & $4.13670932812775 \mathrm{E}-40$ \\
\hline HBM & & 5 & 2.99888096001969 & $1.19842575187367 \mathrm{E}-33$ & $2.61996885836031 \mathrm{E}-101$ \\
\hline ADM & & 5 & 2.99888096001969 & $1.19842575187367 \mathrm{E}-33$ & $2.61996885836031 \mathrm{E}-101$ \\
\hline KHM1 & 100 & 5 & 2.99888096001969 & $1.19842575187367 \mathrm{E}-33$ & $2.61996885836031 \mathrm{E}-101$ \\
\hline KHM2 & & 5 & 2.99888096001969 & $1.19842575187367 \mathrm{E}-33$ & $2.61996885836031 \mathrm{E}-101$ \\
\hline KHM3 & & 5 & 2.99888096001969 & $1.19842575187367 \mathrm{E}-33$ & $2.61996885836031 \mathrm{E}-101$ \\
\hline KHM4 & & 5 & 2.99888096001969 & $1.19842575187367 \mathrm{E}-33$ & $2.61996885836031 \mathrm{E}-101$ \\
\hline
\end{tabular}




\section{References}

[1] M.A.Noor and K.I.Noor, Improved iterative methods for solving nonlinear equations, Appl.Math.Comput.183, (2006), 774-779.

[2] M.J.Emamzadeh and M.T.Kajani, Nonlinear Fredholm Integral Equation of the Second Kindwith Quadrature Methods ,Journal of Mathematical Extension,Vol. 4, No. 2 (2010), 51-58.

[3] S. Abbasbandy, Extended Newton's method for a system of nonlinear equations by modifiedAdomian decomposition method, Appl. Math. Comput.170, (2005), 648-6567.

[4] H. jafari and V.D.Gejji, Revised Adomian decomposition method for solving a system of nonlinear equations, Appl.Math.Comput.175, (2006), 1-7.

[5] F.Awawdeh, On new iterative method for solving systems of nonlinear equations. Springer, DOI10.1007/s11075-009-9342-8.

[6] A.R.Vahidi, Sh. Javadi and S.M. Khorasani, Solving System of Nonlinear Equations by restartedAdomain,s method. Appl. Math. Comput. 6 (2012), 509 -516.

[7] A.R. Soheili, S.A. Ahmadian and J. Naghipoor, A Family of Predictor-Corrector Methods Basedon Weight Combination of Quadratures for Solving Nonlinear Equations, ISSN 17493889, 6 (2008) 29-33 IJNS. (2008).08.15/159.

[8] P. Wang, A Third-Order Family of Newton-Like Iteration Methods for Solving Nonlinear Equations Journal of Numerical Mathematics and Stochastics,3 (1) : 13-19, 2011 ,Online: ISSN2151-2302.

[9] M.A. Noor and K.I. Noor, Improved iterative methods for solving nonlinear equations, Appl. Math. Comput. 183, (2006), 774-779.

[10] C. Chun, A new ityerative method for solving nonlinear equations, Appl.Math.Comput.178(2), (2006), 415-422.

[11]Y.L.Kim, C.Chun and W.Kim, Some Third-order Curvature Based Methods for Solving Nonlinear Equations, Studies in Nonlinear Sciences 1 (3): 72-76, 2010.

[12] M. M. Hosseini and B. Kafash, An Efficient Algorithm for Solving System of Nonlinear Equations, Appl. Math. Sci., 4 (2010), 119 - 131.

[13] M.T. Darvishi and Byeng-Chun Shin, High-Order Newton-Krylov Methods to Solve Systems of Nonlinear Equations. J.KSIAM 15 (2011),19-30.

[14] M.T. Darvishi, a two-step high order newton-like method for solving systems of nonlinear equations, International Journal of Pure and Applied Mathematics, 57 (2009), 543-555

[15] M.A.Hafiz, M.S.M. Bahgat, Modified of Householder iterative method for solving nonlinear system of equations. J. Mathematical and Computational Science (2012) (Under Publication) .

[16] M.A.Hafiz, M.S.M. Bahgat, An Efficient Two-Step Iterative Method for Solving System of Nonlinear Equations. Journal of Mathematics Research; 4, (4), (2012) 28-34

[17] M. A. Noor, M. Waseem, K.I. Noor, E. Al-Said, Variational iteration technique for solving a system of nonlinear equations. Optim Lett, (2012), DOI 10.1007/s11590-012-0479-3.

[18] M.A. Noor, M.Waseem, Some iterative methods for solving a system of nonlinear equations, Computers and Mathematics with Applications 57 (2009) 101-106.

[19] M. T. Darvishi and A. Barati, A third-order Newton-type method to slove systems of nonlinear equations, Appl. Math. Comput.187, (2007), 630-635.

[20] M. T. Darvishi and A. Barati, Super cubic iterative methods to solve systems nonlinear equations, Appl. Math. Comput.188, (2007), 1678-1685.

[21] J.H.He, Homotopy perturbation technique, Comput. Methods Appl. Mech. Eng. 178(3-4), (1999), 257-262.

[22] A. Golbabai, M. Javidi, A new family of iterative methods for solving system of nonlinear algebric equations, Appl. Math. Comput.190, (2007), 1717-1722.

[23] M. Ozel: A New Decomposition Method For Solving System Of Nonlinear Equations. Appl. Math. Comput. 15 (2010), 89-95.

[24] J.R. Sharma and R. Sharma, Some Third Order Methods for Solving Systems of Nonlinear Equations, World Academy of Science, Engineering and Technology 602011. 
[25] J.L. Hueso, E.Martines and J.R.Torregrosa, Third order iterative methods free from second derivative for nonlinear systems, Applied Mathematics and Computation 215 (2009) 58-65.

[26] R. L.Burden and J. D. Farires,"Numerical Analysis". 9rd ed. Boston, USA, (2011).

[27] M.Podisuk, U. Chundang and W. Sanprasert, Single step formulas and multi-step formulas of the integration method for solving the initial value problem of ordinary differential equation, Applied Mathematics and Computation 190 (2007) 1438-1444.

[28] M.G.Sánchez, Á.Grau and J.L.Díaz-Barrero, on computational order of convergence of some multi-precision solvers of systems of nonlinear equations, arXiv:1106.0994v1 [math.NA] 6 Jun 2011.

[29] H.H.Homeier, On Newton-type methods with cubic convergence, Journal of Computational and Applied Mathematics 176 (2005) 425-432.

[30] M.A.Hafiz, M.S.M. Bahgat, extended and modified Halley's iterative method for solving nonlinear system of equations. J. Mathematical and Computational Science (2012) (Under Publication). 